\title{
GCU
}

Glasgow Caledonian

University

University for the Common Good

\section{The computer nose best}

Jilani, Shelina; Ugail, Hassan; Logan, Andrew

Published in:

2019 13th International Conference on Software, Knowledge, Information Management and Applications (SKIMA

DOI:

10.1109/SKIMA47702.2019.8982474

Publication date:

2020

Document Version

Author accepted manuscript

Link to publication in ResearchOnline

Citation for published version (Harvard):

Jilani, S, Ugail, H \& Logan, A 2020, The computer nose best. in 2019 13th International Conference on Software, Knowledge, Information Management and Applications (SKIMA. 2019 13th International Conference on Software, Knowledge, Information Management and Applications, SKIMA 2019, IEEE. https://doi.org/10.1109/SKIMA47702.2019.8982474

\section{General rights}

Copyright and moral rights for the publications made accessible in the public portal are retained by the authors and/or other copyright owners and it is a condition of accessing publications that users recognise and abide by the legal requirements associated with these rights.

Take down policy

If you believe that this document breaches copyright please view our takedown policy at https://edshare.gcu.ac.uk/id/eprint/5179 for details of how to contact us. 


\section{The Computer Nose Best}

\author{
Shelina Jilani* \\ Centre of Visual Computing \\ University of Bradford \\ Bradford, UK. \\ S.Jilani@bradford.ac.uk
}

\author{
Hassan Ugail \\ Centre of Visual Computing \\ University of Bradford \\ Bradford, UK. \\ h.ugail@bradford.ac.uk
}

\author{
Andrew Logan \\ Department of Vision Sciences \\ Glasgow Caledonian University \\ Glasgow, Scotland. \\ Andrew.Logan@gcu.ac.uk
}

\begin{abstract}
The nose is the most central feature on the face which is known to exhibit both gender and ethnic differences. It is a robust feature, invariant to expression and known to contain depth information. In this paper we address the topic of binary ethnicity classificiation from images of the nose, using a novel dataset of South Asian, Pakistani images. To the best of our knowledge, we are one of the first to attempt demographic (ethnicity) based identification based solely on information from the nose.
\end{abstract}

A two-category (Pakistani vs Non-Pakistani) task was used in combination with Deep learning (ResNet) based and VGG-based pre-trained models. A series of experiments were conducted using ResNet-50, ResNet-101, ResNet-152, VGG-Face, VGG-16 and VGG-19, for feature extraction and a Linear Support Vector Machine for classification. The experimental results demonstrate ResNet-50 achieves the highest performance accuracy of $94.1 \%$. In comparison, the highest score for the VGG-based models (VGG-16) was $90.8 \%$. These results demonstrate that information from the nose is sufficient for deep learning models to achieve $\mathbf{9 0 \%}$ accuracy on judgements of ethnicity.

Keywords-Deep Residual Neural Networks; Ethnicity; Nose; Pakistani Face Database; Human Attributes; Feature Extraction.

\section{INTRODUCTION}

Ethnic variations in facial appearance are significant: the physiological makeup is dependent upon a person's ethnicity [1] [2]. In the field of computer vision, it is well established that facial features are a critical source of information. Facial recognition systems rely on the attributes of the face to aid identification, which is why it is one of the most studied research areas. And, although the face provides demographic information relating to age, gender and ethnicity, the latter remains the most invariant trait for image-based classification tasks.

The application of ethnicity-specific information can assist in the field of security and surveillance, as a way of either; (1) identifying a suspect or (2) filtering amongst nominals. The identification of criminals through facial morphology has a long history thanks to Alphonse Bertillon [3] and Salvatore Ottolenghi [4]. More so, the implementation of ethnicityspecific information can be used to increase the performance of existing image localization algorithms [5].

The recent surge in popularity of deep learning algorithms is due to their increased performance power and reduced computation time [6]. By learning from the most discriminatory features, neural network methods have been used to address tasks relating to face-ageing [7] smile dynamics [8] and ethnicity classification [9].
There has been a great deal of focus within machine learning literature, on the use of varied datasets for the purpose of ethnicity classification such as facial profiles [10], silhouetted face profiles [11], front-facing facial images [12] [13] [14] and more recently Near-Infrared (NIR) images [15]. However, the implementation of feature-specific ethnicity classification from the nose, presently remains unchallenged. As does the use of data derived from the South Asian, Pakistani population. This paper addresses the task of ethnicity classification from a novel dataset of Pakistani facial feature images.

The human nose is the most centralized and protrusive feature on the face [16]. By using images of the nose as a primary feature for image-based classification, there are several advantages. Firstly, the shape of the nose is robust to changes associated with variations in facial expression. It also is unaffected by facial hair. Moreover, anthropometric studies report both gender and ethnicity differences in nose shape and size [17] [18] [19]. The stability of nose appearance within an individual, and the invariance of nasal shape over time, highlights the importance of this surface feature for identification.

In this paper, we focus on ethnicity as a demographic trait, visible within the nose, and conduct a series of experiments using a range of pre-trained machine learning algorithms which are Residual Neural Network (ResNet) and VGG-Face based. The pre-trained models used as part of our proposed methodology are: (1) VGG-Face, (2) VGG-16 and (3) VGG19. While, the Residual Neural Network (ResNet) used are; (1) ResNet-50, (2) ResNet-101 and (3) ResNet-152.

For the research presented here, we define ethnicity as a person's cultural and ancestral background. Images of the nose which were cropped from full-face images were classed as either Pakistani or Non-Pakistani, based on a specific selection criterion, which is discussed in the upcoming feature database section.

The proposed method for ethnicity classification consists of 2 components; (i) Feature extraction using weights of VGGFace, VGG-16, VGG-19, ResNet-50, ResNet-101 and ResNet-152 and (ii) ethnicity classification using a linear Support Vector Machine (SVM) algorithm as a binary procedure.

The outline of the paper is follows; section II presents a literature review of previous studies on the use of demographic (ethnicity) information within a machine learning framework. In section III a novel dataset of Pakistani 
faces is discussed, which is created exclusively for the experiments presented as part of this paper. In section IV, we discuss the methodology employed for the experiments. In section $\mathbf{V}$ we present the experimental results. Section VI consists of a discussion and finally, we conclude our work in section VIII.

\section{LITERATURE REVIEW}

Over the years, the task of ethnicity classification has been attempted by multiple machine learning algorithms under diverse assignments. Lu et al. [12] used Linear Discriminant Analysis (LDA) to ascertain ethnicity classification from a database of 2,630 images from 263 subjects. They reported a $96.3 \%$ performance accuracy for binary (Asian vs Non-Asian) classification.

Hosoi et al, [13] integrated Gabor Wavelet features and Retina Sampling to obtain the most informative features. A support vector machine was used for the classification of the features into three classes: Asian, African and European. They achieved the following accuracy; $96 \%, 94 \%$ and $93 \%$ respectively.

Roomi et al. [20] used the Viola Jones algorithm for face detection after which features such as skin colour, lip colour and normalized forehead region were extracted for racial classification. The three races classified as part of the study were Caucasoid, Negroid and Mongoloid. The authors proposed that the distinction between the three human groups were historically agreed by anthropologists and exhibited differences in head shape, skin colour, lips and hair texture.

For skin colour extraction, the face images were converted from RGB to the YCbCr colour space with each pixel being classed as either 'skin' or 'non-skin.' Feature extraction was carried out by projecting edge maps using Sobel edge detection. On a dataset created from the Yale and FERET image database, the authors reported an accuracy of $81 \%$ for the Caucasoid, Negroid and Mongoloid races.

Yewenberg et al. [21] investigated the classification of ethnicity in addition to traits such as age, gender and emotion on the Face Attributes Dataset (FAD). The authors conducted baseline experiments using a pre-trained CNN model; AlexNet and compared the results with their novel landmark augmented CNN (LACNN). The tested ethnicity (White) was accurately classified using AlexNet with $82.7 \%$ accuracy, whereas LACNN marginally increased the performance accuracy to $83.35 \%$. The research highlighted the strength of $\mathrm{CNN}$ architectures and the effectiveness of using a large and varied dataset.

While the task of ethnicity classification has been addressed using images of the entire face. There is a handful of literature, which has investigated the robustness of facial features such as the eyes and the nose, for ethnicity classification. For example, Qiu et al. [22] [22] suggested a model to determine ethnicity (Asian vs Non-Asian) from iris images.
Comparably, the nose has also been shown to be an efficient biometric [23]. Although the availability of nose-related literature is limited.

LV et al. [24] introduced a novel 3D Nose Shape Net classifier for gender and ethnicity classification. To construct a 3D Nose Shape Net, a nose measurement method is required to ascertain the distances between the different noses within a given dataset and then the images are clustered into groups. By using the nose clustering results, the $3 \mathrm{D}$ nose is constructed. Using the Bosphorus3D face image database and the FRGC2.0, the authors reported an ethnicity classification of $89.2 \%$ for the Asian ethnicity and $87.4 \%$ for the White.

A similar study on the use of 3D nose shape information has also been reported by Drira and colleagues (2009) [25].

Song et al. [26] studied extracting the quantity and position of pores on the skin of the nose as a biometric. A performance accuracy of $88.07 \%$ was reported and the research demonstrated that nose-pores are an encouraging avenue for biometric identification.

The feasibility of image-based nose biometrics for determining identity information was investigated by Zehngut [27]. Two types of experiments were carried out; (1) comparing full-face and nose biometrics using the NIST's Face Recognition Grand Challenge (FRGC) database of 12,776 images and (2) comparing full face and nose biometrics for occluded face images, using the AR Face database of 3,288 images. Discriminative nasal features were extracted using Kernel Class-Dependence Feature Analysis (KCFA) based on Optimal Trade-off Synthetic Discriminant Function (OTSDF) filters.

To conclude, the KCFA features extracted from the nose region were reported to significantly outperform commercial face matching algorithms. Such literature demonstrates the strength of the nose as an information-rich feature. The literature review demonstrates that the use of nose images including aspects of the feature such as nose-pores, for demographic (ethnicity) identification has proven successful. The nose is a robust and discriminative feature and the literature review proves the reliability.

\section{The Pakistani Face Database}

The Pakistani Face Database has been developed by researchers within the University of Bradford, United Kingdom. 100 Pakistani and 100 Non-Pakistani images were selected from the Pakistani face database. Images which constitute the non-Pakistani class, are varied and include Black, Caucasian, East Central Asian, Kurds etc. as well as the South Asian, Gujarati (Indian) ethnicity. The ethnicity of each the image was determined by asking the participants to self-identify their ethnicity. For participants of Pakistani origin, eligibility was dependant on whether both the maternal and paternal parents were of Pakistani ethnicity.

Images were taken using a custody capture system, Halo [10] which utilises machine vision cameras to capture high quality face images, with balanced lighting. All the facial images 
were cropped around the region of the nose using Photoshop CS6 to generate a sub-dataset of the varied nose regions, see fig. 1. To keep all the information, it was ensured that the entire nose was cropped, showing clearing the nasal bridge, the pronasale (nasal tip) as well as the nasal alars (nostrils).
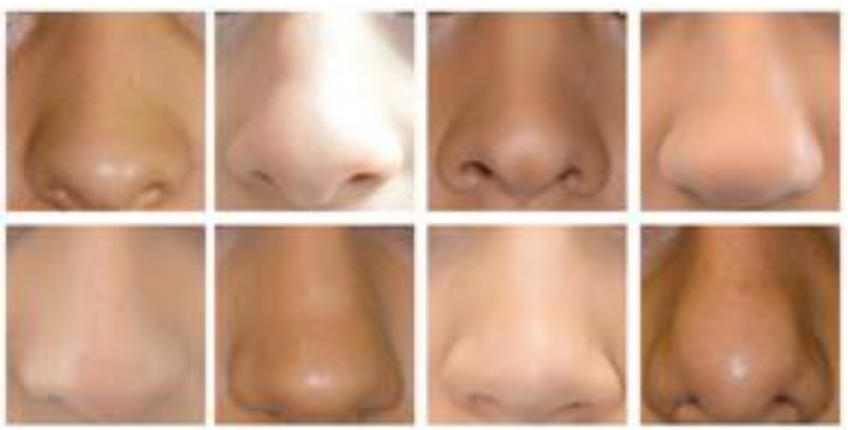

Fig. 1 An example of nose images that have been processed using Adobe Photoshop CS6. (The images used have been reproduced in line with the approved ethics from the University of Bradford, UK and participant consent).

\section{MeTHODOLOGY}

Our approach was to extract nose features using six pretrained machine learning models, three VGG-based and three Deep-learning based. The models used include; VGG-Face, VGG-16, VGG-19, ResNet-50, ResNet-101 and ResNet-152. Followed by a discriminative binary classification using a Linear Support Vector Machine.

A total of eight experiments were conducted on the following datasets: (1) 2,200 isolated nose crops, (2) 1,200 isolated eyes and nose crops, (3) 1,200 isolated nose and mouth crops and (4) 1,200 isolate mouth and eyes crops.

\section{A. Data Pre-Processing}

All images used in our experiments were resized to $224 \times 224$ pixels to ensure they conform to the input criteria of the pretrained models. Furthermore, data augmentation was carried out for the training data using rotations at the degree of $10^{\circ}$ right, $10^{\circ}$ left, $90^{\circ}, 180^{\circ}$ and $270^{\circ}$ as well as random crops. This led to a minimum of a twelvefold increase in the original data size.

\section{B. Feature Extraction}

The activation of the last pooling layer of each of the ResNet models was used, for feature representation.

In total, three sets of features were retrieved using ResNet50, RestNet-101, and ResNet-152 Neural Networks, per experiment. We decided to exclude the last output layer of the Fully Connected layer (FC), since it was trained on a set of different data (i.e. objects) compared to the facial features which are presently used. Additionally, research proves that the lower layers of the Deep Neural Network are adequate in learning generic features $[23,28]$.
Comparably, for the VGG feature representation using the VGG-Face, VGG-16 and VGG-19 models, we used the middle Fully Connected layer (FC7) and discounted FC6 and FC8.

\section{Classification}

Having used machine learning-based feature extraction with VGG-Face, VGG-16, VGG-19, ResNet-50, ResNet-101 and ResNet-152 models, independently. A linear classifier was employed for a two-category ethnicity classification.

Support Vector Machine (SVMs) are supervised machine learning models that function to identify a hyperplane, which best classifies data points within a given data space. Published literature has determined that SVMs is a powerful binary classifier which operates by defining an optimum separating hyperplane between two classes of data [29] [30].

In our work, the two classes are Pakistani and Non-Pakistani. The SVM algorithm classifies data by defining an optimum separating hyperplane between two data classes, which typically involves solving an optimization problem.

Given a training set,

$$
\left\{\left(x_{i}, y_{i}\right)\right\}_{1 \leq i \leq n}, x_{i} \in \mathbb{R}^{d}, y_{i} \in\{+1,-1\},
$$

SVM finds the Optimum Separating Hyperplane (OSH) by solving,

$$
\left\{\begin{array}{lr}
\min _{w, b} & \frac{1}{2}\|w\|^{2} \\
\text { with } & y_{i} \cdot(w \cdot x+b) \geq 1
\end{array}\right.
$$

for $i=1 \ldots n$ are the observations, where the weights $w$ and $b$ the bias is learned during training. The classifier is learned such that, $y_{i} \in\{+1,-1\},+1$ denotes images labelled as Pakistani and -1 denotes Non-Pakistani images.

A $k$-fold cross validation technique was employed to evaluate the performance of the pre-trained models.

\section{EXPERIMENTAL RESULTS}

By extracting features from images using three pretrained deep-learning models; ResNet-50, ResNet-101 and ResNet-152, we achieved results above $90 \%$ for the binary classification of a Pakistani nose on a dataset of 2,200 nose images, (experiment 1) see table I.

TABLE I. PERFORMANCE ACCURACY FOR THE BINARY CLASSIFICATION OF PAKISTANI NASAL IMAGES USING RESIDUAL NEURAL NETWORKS.

\begin{tabular}{|c|c|}
\hline $\begin{array}{c}\text { Feature } \\
\text { Extraction } \\
\text { Model }\end{array}$ & \begin{tabular}{c} 
Classifier \\
\cline { 2 - 2 } ResNet-50
\end{tabular} \\
\hline ResNet-101 & $\mathbf{9 4 . 1 \%}$ \\
\hline MesNet-152 & $93.4 \%$ \\
\hline
\end{tabular}


In contrast, the experiments results using the three VGGbased models namely; VGG-Face, VGG-16 and VGG-19 are varied between each other and are essentially, lower when compared to the ResNet models. The performance accuracies are directly comparable to the ResNet models and demonstrate the finding that the accuracy is poorer with VGG than ResNet. This may be attributable to the finding that the ResNet modes are classed as deep learning models, which are architecturally different and report to yield higher accuracies. However, VGG-16 does outperform VGG-F and VGG-19 in the binary classification task, see table II.

TABLE II. PERFORMANCE ACCURACY FOR THE BINARY ClassifiCATION OF PAKISTANI NASAL IMAGES USING VGG-BASED MODELS.

\begin{tabular}{|c|c|}
\hline $\begin{array}{c}\text { Feature } \\
\text { Extraction } \\
\text { Model }\end{array}$ & $\begin{array}{c}\text { Classifier } \\
\text { Manear Support Vector } \\
\text { Machine (SVM) }\end{array}$ \\
\hline VGG-16 & $\mathbf{9 0 . 8 \%}$ \\
\hline VGG-19 & $87.5 \%$ \\
\hline VGG-Face & $79.9 \%$ \\
\hline
\end{tabular}

Additional experiments were carried out using isolated face feature crops, to ascertain whether feature combinations impacted performance accuracy. The feature combinations used consisted of; (1) eyes and nose, (2) nose and mouth and (3) mouth and eyes, see table III.

TABLE III. ACCURACY FOR THE BINARY CLASSIFICATION OF FACEFEATURE COMBINATION

\begin{tabular}{|c|c|c|c|}
\hline \multirow{2}{*}{$\begin{array}{c}\text { Feature } \\
\text { Extraction } \\
\text { Models } \\
\end{array}$} & \multicolumn{3}{|c|}{ Linear Support Vector Machine (SVM) } \\
\hline & $\begin{array}{c}\text { Eyes \& } \\
\text { Nose }\end{array}$ & Nose \& Mouth & Mouth \& Eyes \\
\hline ResNet-50 & $85.8 \%$ & $91.8 \%$ & $87.8 \%$ \\
\hline ResNet-101 & $85.5 \%$ & $90.5 \%$ & $88.8 \%$ \\
\hline ResNet-152 & $84.4 \%$ & $90.2 \%$ & $84.8 \%$ \\
\hline VGG-16 & $81.5 \%$ & $85.2 \%$ & $82.8 \%$ \\
\hline VGG-19 & $79.5 \%$ & $80.3 \%$ & $77.6 \%$ \\
\hline VGG-Face & $72.6 \%$ & $75.8 \%$ & $73.6 \%$ \\
\hline
\end{tabular}

The results achieved from the isolated feature combinations demonstrate that, on average, the nose and mouth features yield the highest results $(85.63 \%)$. Moreover, for all the tested pre-trained models, the nose and mouth feature combination consistently yield the highest performance accuracy.

The results are closely followed by the mouth and eyes and the eyes with nose combination with $82.56 \%$ and $81.55 \%$ average accuracies, respectively. The results show that the internal features i.e. eyes, nose and mouth provide reliable information to separate between the Pakistani and NonPakistani faces.

Importantly, the reported results are in line with literature which proposes that the eyes, nose and mouth are important in determining ethnicity in humans [13]. To determine the effectiveness of the results achieved, performance metrics such as sensitivity and specificity were calculated. Sensitivity is the value of positive cases classified correctly (TPR), and specificity is the value of negative cases correctly rejected (TNR) [31]. By combining sensitivity and specificity the overall performance accuracy of the classification algorithm is reported. A visual representation of the values is shown in form of a Receiver Operating Characteristics (ROC) Graph.

Fig. 2 shows the ROC generated for the nose image experiment using the three tested deep learning algorithms; ResNet-50, ResNet-101, ResNet-152 (experiment 1). While, figure 3 shows the ROC for the VGG-based models namely VGG-Face, VGG-16 and VGG-19 models (experiment 2).

By looking at fig. 2 it is evident that all three models performed similarly (i.e. within the region of $>90 \%$ ), which is depicted by the position of the performance lines, that are positioned to the left of the axis.



Fig. 2 Receiver Operating Characteristics (ROC) Curve for the binary classification of the Pakistani nose, using Residual Neural Networks (ResNet-50, ResNet-101 and ResNet-152). The True Positive Rate (TPR) is the value of cases where the data has been correctly identified. False Positive Rate (FPR) is the value of cases where data has been incorrectly identified as a positive.

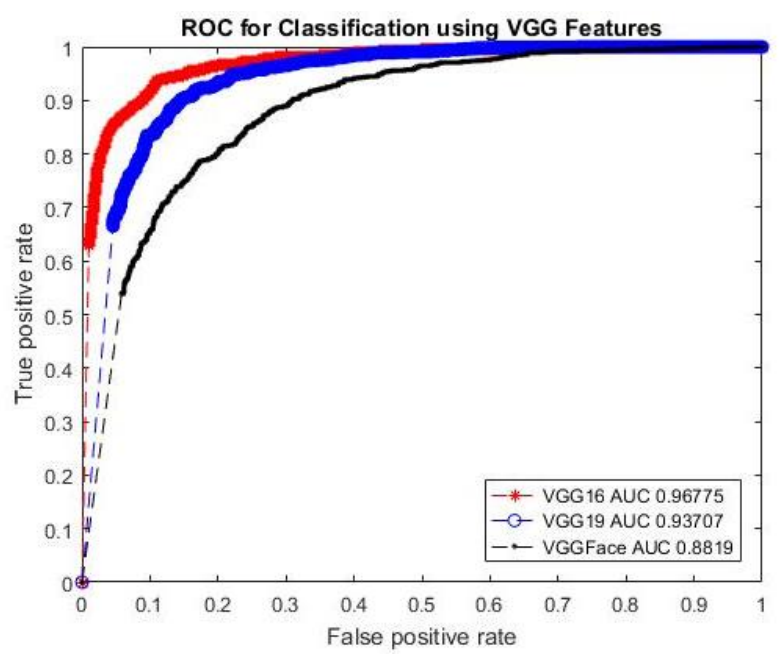

Fig. 3 Receiver Operating Characteristics (ROC) Curve for the binary classification of the Pakistani nose, using VGG-based pre-trained models, namely VGG-16, VGG-19 and VGG-Face. The True Positive Rate (TPR) is the value of cases where the data has been correctly identified. False Positive Rate (FPR) is the value of cases where data has been incorrectly identified as a positive. 
Despite having seemingly lower accuracy, RestNet101, having an area under the curve (AUC) of 0.9773 demonstrates competitive performance. This shows that even with limited facial detail i.e. nose images only, the deeplayered architecture is sufficient at capturing ethnicity traits.

Fig. 3 shows the ROC for the isolated nose images using VGG-features. Unlike the ResNet models which are trained on none-face images and performed competitively. The VGG-based models, which are trained specifically on a dataset of faces, did not perform comparably to the ResNet models. However, it is apparent from the ROC curve that VGG-16 outperforms the other two models, and VGG-19 outclassed VGG-Face. VGG-16 is a deep Neural Network model developed by the University of Oxford. In total, the model consists of 37 layers, 13 of which are convolutional layers and 3 Fully Connected (FC) layers [32]. Such results are surprising especially since they are trained on aspects of the face.

Comparing both the ROC curves, deep neural networks extract relevant information, especially when there is very little facial detail, to yield a high-performance accuracy, in comparison to the VGG-based models.

\section{DISCUSSION}

We investigated the distinctiveness of the nose as a marker of ethnicity for the South Asian, Pakistani population. The results show that deep learning algorithms outperform VGG-based models, on the classification of isolated face feature images. The results presented within this paper advance current understanding of ethnicity determination because, to the best our knowledge, the application of deep learning algorithms for the ethnic classification of Pakistani faces based on a single feature, specifically the nose, has not previously been attempted. Moreover, there is limited evidence available on the application of the nose as biometric feature for identification.

As per table 1, ResNet-50 achieved a performance accuracy of $94.1 \%$, followed closely by ResNet-152 and ResNet-101 with classification at $93.6 \%$ and $93.4 \%$ respectively. The closeness in performance between the ResNet models is consistent with results presented by $\mathrm{He}$ et al., [33] The 50/101/152-layer ResNets yield high and accurate results given the considerably increased depth of the architectures.

Due to the novelty of our stimuli (i.e. isolated nose database) and the specificity of our approach (ethnicity determination based on nose information only), the opportunity to compare our results with previous reports is limited. Nonetheless, our results suggest greater accuracy than those presented by LV et al. [24] who utilized 3D nose shape classifiers and reported an ethnicity classification of $89.2 \%$ for the Asian and $87.4 \%$ for the White ethnic group.

Further, our results support the proposal that the nose is a useful feature for determining Pakistani ethnicity. This is in agreement with findings from Zehngut [27] who reported a machine learning based experiment on the distinctiveness of the nose as a feature. While, the authors did not investigate ethnicity classification, the research highlights the value of the nose. Importantly, it is acknowledged that the Pakistani Face Database is predominantly collected in the city of Bradford and the Pakistani population it houses may be homogenous in terms of the geographical area of Pakistan from which they originate. This is an inherent limitation and uncontrollable unless a larger dataset is created, constituting different regions of Pakistan.

To the best of our knowledge, the nose as an isolated feature to address a binary ethnicity classification task has not been previously conducted, using either ResNet or VGG-features. The results achieved from all 8 experiments establish that deep learning algorithms outperform VGG-based models. We suggest that this is due to their deep architecture. Further, given the diversity and sexual dimorphism associated with the nose, in situations where humans may struggle to classify the ethnicity of a face and discriminate between Pakistani and non-Pakistani noses, deep learning methods prove competent in providing information, which is not available from the human visual system.

\section{CONCLUSION}

We addressed the ethnic classification of physical face features, especially the nose. A binary classification task was investigated using a novel dataset of nose images for the South Asian, Pakistani ethnic group.

ResNet and VGG-features were extracted and fed to a Linear Support Vector Machine (SVMS) classifier. A performance accuracy of $94.1 \%$ was concluded for ResNet-50, whereas VGG-16 classified nose images with $90.8 \%$ accuracy. Our results are novel since ethnic classification of the Pakistani nose has not been previously attempted.

As part of future work, we suggest using partial feature data to attempt the challenge of ethnicity classification. Moreover, while SVM is a robust binary classifier, we suggest using a different classification model such as Decision Trees and boosting Decision Trees. By implementing the Decision Trees classifier for supervised learning, the two-class ethnicity task can be attempted on nose data, and compared to the robustness of our current framework.

\section{REFERENCES}

[1] Islam, M.T., Workman, S., Wu, H., Jacobs, N. and Souvenir, R., 2014, March. Exploring the geo-dependence of human face appearance. In IEEE Winter Conference on Applications of Computer Vision (pp. 1042-1049). IEEE.

[2] Greenwell, C., Spurlock, S., Souvenir, R. and Jacobs, N., 2014, November. GeoFaceExplorer: Exploring the geo-dependence of facial attributes. In Proceedings of the 3rd ACM SIGSPATIAL International Workshop on Crowdsourced and Volunteered Geographic Information (pp. 32-37). ACM.

[3] Galton, F., 1896. Signaletic Instructions, including the Theory and Practice of Anthropometrical Identification. Nature, 54(1407), p.569.

[4] Ottolenghi, S. and von Borosini, V., 1913. The Scientific Police. Journal of the American Institute of Criminal Law and Criminology, pp.876-880.

[5] Zamir, A.R. and M. Shah, Image geo-localization based on multiplenearest neighbor feature matching usinggeneralized graphs. IEEE transactions on pattern analysis and machine intelligence, 2014. 36(8): p. 1546-1558. 
[6] Ranjan, R., et al., Deep learning for understanding faces: Machines may be just as good, or better, than humans. IEEE Signal Processing Magazine, 2018. 35(1): p. 66-83.

[7] Elmahmudi, A. and Ugail, H., 2019. The Effect of FaceAgeing in Face Recognition (No. 809). EasyChair.

[8] Ugail, H. and A.A.A. Aldahoud, The Biometric Characteristics of a Smile, in Computational Techniques for Human Smile Analysis. 2019, Springer. p. 47-56.

[9] Jahandideh, R., A.T. Targhi, and M. Tahmasbi, Physical Attribute Prediction Using Deep Residual Neural Networks. arXiv preprint arXiv:1812.07857, 2018.

[10] Jilani, S.K., Ugail, H., Bukar, A.M., Logan, A. and Munshi, T., 2017, September. A Machine Learning Approach for Ethnic Classification: The British Pakistani Face. In 2017 International Conference on Cyberworlds (CW) (pp. 170-173). IEEE.

[11] Tariq, U., Hu, Y. and Huang, T.S., 2009, November. Gender and ethnicity identification from silhouetted face profiles. In 2009 16th IEEE International Conference on Image Processing (ICIP) (pp. 24412444). IEEE.

[12] Lu, X. and Jain, A.K., 2004, April. Ethnicity identification from face images. In Proceedings of SPIE (Vol. 5404, pp. 114-123).

[13] Hosoi, S., Takikawa, E. and Kawade, M., 2004, May. Ethnicity estimation with facial images. In Sixth IEEE International Conference on Automatic Face and Gesture Recognition, 2004. Proceedings. (pp. 195-200). IEEE.

[14] Guo, G. and Mu, G., 2013, April. Joint estimation of age, gender and ethnicity: CCA vs. PLS. In 2013 10th IEEE International Conference and Workshops on Automatic Face and Gesture Recognition (FG) (pp. 1-6). IEEE.

[15] Narang, N. and Bourlai, T., 2016, June. Gender and ethnicity classification using deep learning in heterogeneous face recognition. In 2016 International Conference on Biometrics (ICB) (pp. 1-8). IEEE.

[16] Harkema, J.R., S.A. Carey, and J.G. Wagner, The nose revisited: a brief review of the comparative structure, function, and toxicologic pathology of the nasal epithelium. Toxicologic pathology, 2006. 34(3): p. 252-269.

[17] Ozdemir, F. and A. Uzun, Anthropometric analysis of the nose in young Turkish men and women. Journal of Cranio-Maxillofacial Surgery, 2015. 43(7): p. 1244-1247.

[18] Ohki, M., K. Naito, and P. Cole, Dimensions and resistances of the human nose: racial differences. The Laryngoscope, 1991. 101(3): p. 276-278.

[19] Jilani, S.K., H. Ugail, and A. Logan, Inter-Ethnic and Demic-Group Variations in Craniofacial Anthropometry: A Review. PSM Biological Research, 2019. 4(1): p. 6-16.

[20] Roomi, S.M.M., Virasundarii, S.L., Selvamegala, S., Jeevanandham, S. and Hariharasudhan, D., 2011, December. Race classification based on facial features. In 2011 third national conference on computer vision, pattern recognition, image processing and graphics (pp. 5457). IEEE

[21] Lewenberg, Y., Bachrach, Y., Shankar, S. and Criminisi, A., 2016, March. Predicting personal traits from facial images using convolutional neural networks augmented with facial landmark information. In Thirtieth AAAI Conference on Artificial Intelligence.

[22] Qiu, X., Sun, Z. and Tan, T., 2006, January. Global texture analysis of iris images for ethnic classification. In International Conference on Biometrics (pp. 411-418). Springer, Berlin, Heidelberg.

[23] Chang, K.I., K.W. Bowyer, and P.J. Flynn, Multiple nose region matching for $3 D$ face recognition under varying facial expression. IEEE Transactions on Pattern Analysis and Machine Intelligence, 2006. 28(10): p. 1695-1700.

[24] Lv, C., Wu, Z., Zhang, D., Wang, X. and Zhou, M., 2018. 3D Nose shape net for human gender and ethnicity classification. Pattern Recognition Letters.

[25] Drira, H., Amor, B.B., Daoudi, M. and Srivastava, A., 2009, June. Nasal region contribution in $3 \mathrm{D}$ face biometrics using shape analysis framework. In International Conference on Biometrics (pp. 357-366). Springer, Berlin, Heidelberg.

[26] Song, S., Ohnuma, K., Liu, Z., Mei, L., Kawada, A. and Monma, T., 2009. Novel biometrics based on nose pore recognition. Optical Engineering, 48(5), p.057204.

[27] Zehngut, N., Juefei-Xu, F., Bardia, R., Pal, D.K., Bhagavatula, C. and Savvides, M., 2015, September. Investigating the feasibility of imagebased nose biometrics. In 2015 IEEE international conference on image processing (ICIP) (pp. 522-526). IEEE.

[28] Karpathy, A., Toderici, G., Shetty, S., Leung, T., Sukthankar, R. and Fei-Fei, L., 2014. Large-scale video classification with convolutional neural networks. In Proceedings of the IEEE conference on Computer Vision and Pattern Recognition (pp. 1725-1732).

[29] Chierchia, G., Pustelnik, N. and Pesquet, J.C., 2016, September. Random primal-dual proximal iterations for sparse multiclass SVM. In 2016 IEEE 26th International Workshop on Machine Learning for Signal Processing (MLSP) (pp. 1-6). IEEE.

[30] Nalavade, K. and Meshram, B.B., 2012. Data Classification Using Support Vector Machine. In National Conference on Emerging Trends in Engineering \& Technology (VNCET) (Vol. 2, pp. 181-184).

[31] Powers, D.M., 2011. Evaluation: from precision, recall and F measure to ROC, informedness, markedness and correlation.

[32] Simonyan, K. and A. Zisserman, Very deep convolutional networks for large-scale image recognition. arXiv preprint arXiv:1409.1556, 2014.

[33] He, K., Zhang, X., Ren, S. and Sun, J., 2016. Deep residual learning for image recognition. In Proceedings of the IEEE conference on computer vision and pattern recognition (pp. 770-778). 